\title{
A rare case of Crohn's ileitis in a patient with constitutional mismatch repair deficiency
}

\author{
Pavlos Kaimakliotis ${ }^{a}$, Francis Giardiello ${ }^{a}$, Ogechukwu Eze $^{\mathrm{b}}$, Brindusa Truta ${ }^{\mathrm{a}}$
}

Johns Hopkins University, School of Medicine, USA

\begin{abstract}
Constitutional mismatch repair deficiency (CMMRD), a variant of Lynch syndrome, is a rare disease characterized by café-au-lait spots, oligopolyposis, glioblastoma and lymphoma. A 24-yearold male, under surveillance for CMMRD, developed Crohn's ileitis after total colectomy with end ileostomy for colorectal cancer and failed to respond to oral corticosteroids. The patient underwent induction and maintenance of remission with vedolizumab infusions. We report the first patient with CMMRD developing Crohn's disease. The choice of immunosuppressive therapy in these patients is challenging and needs to be made according to their risk for malignancy.
\end{abstract}

Keywords Crohn's disease, familial cancer, vedolizumab, constitutional mismatch repair deficiency

Ann Gastroenterol 2017; 30 (3): 1-3

\section{Introduction}

Crohn's disease is a chronic inflammatory bowel disease that can affect any site of the gastrointestinal tract. There are several studies of patients with Crohn's and Lynch syndrome, perhaps suggesting an association between these two conditions [1-4]. A variant of Lynch syndrome is constitutional mismatch repair deficiency (CMMRD). These patients have a biallelic (rather than monoallelic, as seen in Lynch syndrome) germline mutation of one of the mismatch repair genes. This rare, clinically aggressive disorder is characterized by café-au-lait spots and development of a host of malignancies in childhood $[5,6]$.

\section{Case report}

A 24-year-old male from Saudi Arabia with CMMRD syndrome was referred to our clinic for further evaluation of his abdominal pain and high stoma output one year after duodenal segmental resection (D4), for adenocarcinoma.

Departments of ${ }^{a}$ Medicine (Pavlos Kaimakliotis, Francis Giardiello, Brindusa Truta); 'Pathology (Ogechukwu Eze), Johns Hopkins University, School of Medicine, USA

Conflict of Interest: None

Correspondence to: Pavlos Kaimakliotis, MBBS, BSc, Johns Hopkins University, School of Medicine, 733 N Broadway, Baltimore, MD, 21205 USA, Tel.: +1 410955 5000, Fax: +410 614 8510,

e-mail: pkaimak1@jhmi.edu

Received 27 November 2016; accepted 30 January 2017; published online 16 February 2017

DOI: https://doi.org/10.20524/aog.2017.0129
In this patient, CMMRD was caused by a biallelic PMS2 mutation, which led to the development of acute lymphoblastic leukemia at age 3, colorectal cancer (two synchronous lesions) at age 19, and duodenal adenocarcinoma at age 22 years. He had completed multiple courses of chemotherapy throughout his lifetime: POG protocol 9006 with ara-C, prednisone and asparaginase at age 3 for the acute lymphoblastic leukemia, and at age 19 adjuvant mFOLFOX for the colorectal cancer. He had undergone total colectomy with end ileostomy at age 19 for the colorectal cancer. Notably, the patient's parents were first cousins.

Two months before being seen in the clinic, he developed abdominal pain, an 8-kg unintentional weight loss, intermittent nausea and vomiting, and increased stoma output. The patient denied taking non-steroidal anti-inflammatory drugs. Examination revealed a young man of ill appearance weighing $34 \mathrm{~kg}$ (body mass index: $14.7 \mathrm{~kg} / \mathrm{m}^{2}$ ). His abdomen was tender to palpation near the ileostomy site. There were multiple 5-10 mm hypopigmented macules on the trunk and extremities.

Further laboratory investigations revealed the presence of mild microcytic anemia (Hb $10.9 \mathrm{~g} / \mathrm{dL}$ [ref. range: 11.1$15.9 \mathrm{~g} / \mathrm{dL}$ ], MCV $74.9 \mathrm{fL}$ [ref. range: $79-97 \mathrm{fL}$ ]), thrombocytosis $(550 \mathrm{~K} / \mathrm{cu} \mathrm{mm}$ [ref. range: $150-350 \mathrm{~K} / \mathrm{cu} \mathrm{mm}$ ]), elevated fecal calprotectin $(464 \mathrm{mcg} / \mathrm{g}$ [ref. range $<162.9 \mathrm{mcg} / \mathrm{g}$ ]) and C-reactive protein $(2.7 \mathrm{mg} / \mathrm{L}$ [ref. range $<0.5 \mathrm{mg} / \mathrm{L}$ ] $)$. QuantiFERON and stool specimens were negative for tuberculosis, bacterial or parasitic infections (including Clostridium difficile). During ileoscopy we noted patchy erythema and ulcers in the terminal ileum; however, the pathology was consistent with nonspecific acute enteritis. There was no histological evidence of vasculitis, ischemia or cytomegalovirus infection. No granulomata were seen. The patient was treated conservatively for partial small bowel 
obstruction, and discharged on budesonide and mesalamine for suspicion of emerging Crohn's disease.

Two months later, the patient noticed only a minimal improvement of symptoms, despite an increase in the prednisone dose (40 mg daily). Repeat endoscopy showed increased friability of the mucosa, with superficial ulcerations and a dilated ileum with narrowing at the ileostomy. Histological evidence of acute ileitis was present (Fig. 1), but again there were no signs of chronicity. The patient had ostomy revision as his symptoms were likely to be due to partial bowel obstruction (secondary to adhesions) rather than Crohn's disease.

Two months after surgery, symptoms reoccurred. Repeat endoscopy (Fig. 2) revealed mucosal edema and erythema with several aphthoid ulcers. The pathology (Fig. 3) was consistent with chronic ileitis, including pyloric gland metaplasia and moderate crypt distortion, highly suggestive of Crohn's disease.

The findings from the third endoscopy, in combination with the aforementioned histologic findings, as well as the patient's presentation and progression during follow up, led to the final diagnosis of Crohn's disease.

The choice of therapy was complicated by the high underlying risk of malignancy in this patient. Vedolizumab was the drug of choice because of its safety profile. The patient responded well, achieving clinical and endoscopic remission by week 12 . Unfortunately, several months later, surveillance endoscopy revealed jejunal adenocarcinoma, and vedolizumab therapy (6-month therapy duration; 6 infusions total, including induction) was stopped in preparation for surgery. Presently, the patient is recovering well, with ileoscopy scheduled 3 months postoperatively to re-stage his condition. He will continue to undergo surveillance enteroscopy every 6 months.

\section{Discussion}

Several studies have reported the association of Lynch syndrome with Crohn's disease. A recent investigation showed that patients with inflammatory bowel disease and Lynch syndrome are at increased risk of developing colorectal cancer, compared to those with Lynch syndrome alone [1]. To our knowledge, there are no reports of patients with Crohn's and CMMRD, a far more aggressive variant of Lynch syndrome.

In the largest study to date, describing 146 patients from 91 families, 139/146 (95\% of patients) developed one or more cancers [6]. Sixty percent of the families included carried biallelic PMS2 mutations. Common features in these patients included café-au-lait spots, colonic oligopolyposis, nonHodgkin's lymphoma, glioblastoma, and Lynch syndromeassociated tumors (including colorectal cancer and endometrial cancer). Unlike Lynch syndrome, patients with CMMRD who develop colorectal cancer are much younger (median age of 16), and often present with synchronous lesions. Patients with CMMRD require tight adherence to surveillance for the early detection of premalignant tumors [7].

Combination therapy with thiopurines was not considered, given the patient's past medical history and the established risk of cancer with these agents [8]. The development of

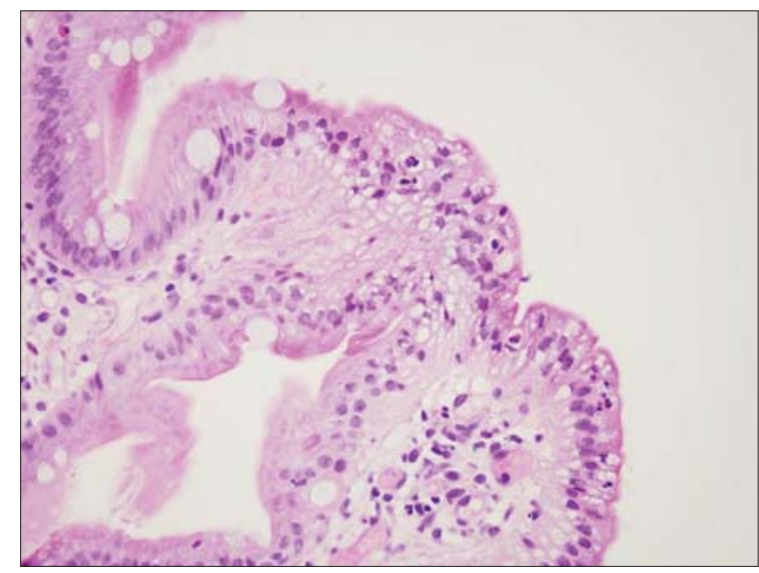

Figure 1 High-power view of a section of the terminal ileum, demonstrating active ileitis. Findings are indicative of acute ileitis, but non-specific for Crohn's disease

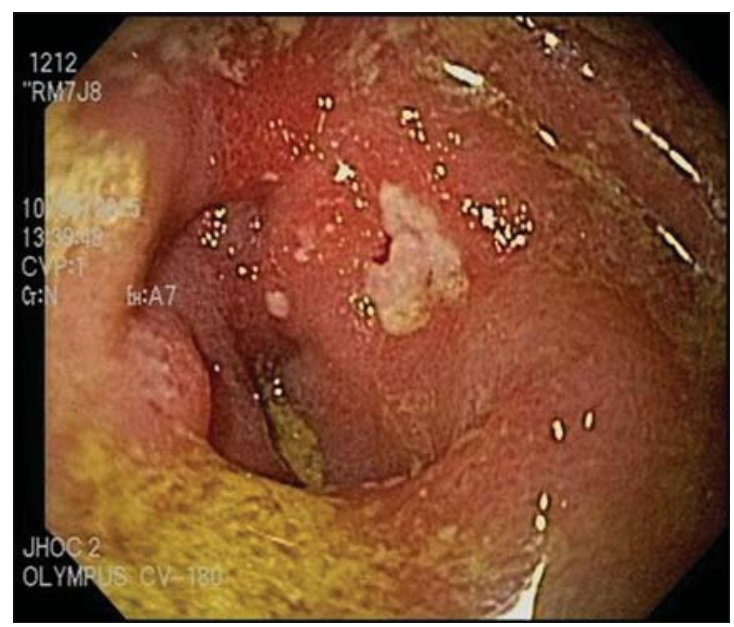

Figure 2 Endoscopic examination of the ileum, demonstrating mucosal edema and erythema with multiple aphthoid ulcers

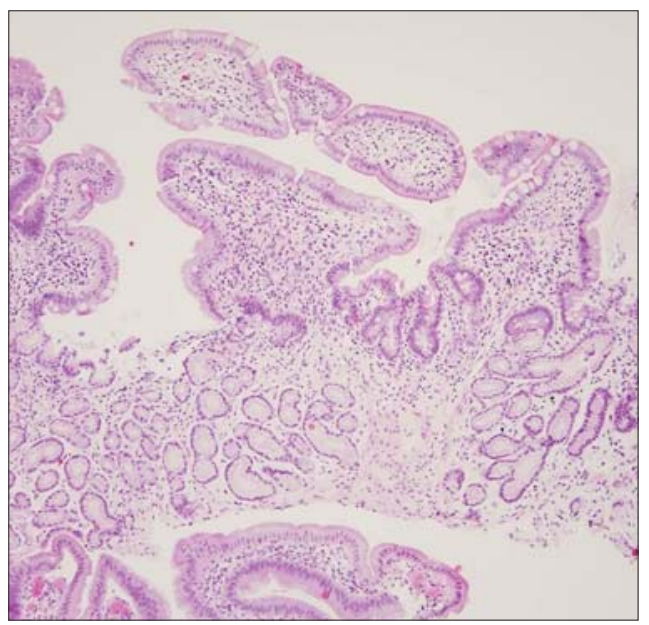

Figure 3 Medium-power view of a section of the terminal ileum, demonstrating active ileitis with signs of chronicity, including crypt architectural distortion and pyloric gland metaplasia. These findings, in combination with the patient's clinical picture, are strongly suggestive of Crohn's disease 
jejunal adenocarcinoma in our patient is unlikely to be related directly to vedolizumab, since he only completed six infusions, although patients receiving over twenty infusions have developed malignancies in previous studies [9].

CMMRD is an exceedingly rare entity, yet the diagnosis should be considered in children and young adults with a strong personal and family history of malignancy. The diagnosis of Crohn's can be difficult to make in certain patients, and this case highlights the importance of histological evidence of disease in making the correct diagnosis.

\section{References}

1. Derikx LA, Smits LJ, van Vliet $S$, et al. Colorectal cancer risk in patients with both Lynch syndrome and inflammatory bowel disease. Clin Gastroenterol Hepatol 2016 Aug 10. doi: 10.1016/j. cgh.2016.08.005. [Epub ahead of print].

2. McNamara KL, Aronson MD and Cohen Z. Is there a role for prophylactic colectomy in Lynch syndrome patients with inflammatory bowel disease? Int J Colorectal Dis 2016;31:9-13.

3. Gentile N, Kane S. Familial adenomatous polyposis, suspected
HNPCC, and Crohn's disease: two cases. Inflamm Bowel Dis 2012;18:E1398.

4. Lourensz K, Jones I. Considerations and management of a patient with three metachronous cancers in association with Lynch syndrome and ileal Crohn's disease: a case report. Int J Surg Case Rep 2015;10:73-75.

5. Trimbath JD, Petersen GM, Erdman SH, Ferre M, Luce MC, Giardiello FM. Cafe-au-lait spots and early onset colorectal neoplasia: a variant of HNPCC? Fam Cancer 2001;1:101-105.

6. Wimmer K, Kratz CP, Vasen HF, et al; EU-Consortium Care for CMMRD (C4CMMRD). Diagnostic criteria for constitutional mismatch repair deficiency syndrome: suggestions of the European consortium 'care for CMMRD' (C4CMMRD). I Med Genet 2014;51:355-365.

7. Vasen HF, Ghorbanoghli Z, Bourdeaut F, et al; EU-Consortium Care for CMMR-D (C4CMMR-D). Guidelines for surveillance of individuals with constitutional mismatch repair-deficiency proposed by the European Consortium "Care for CMMR-D" (C4CMMR-D). J Med Genet 2014;51:283-293.

8. Annese V, Beaugerie L, Egan L, et al; ECCO. European Evidencebased Consensus: Inflammatory Bowel Disease and Malignancies. J Crohns Colitis 2015;9:945-965.

9. Colombel JF, Sands BE, Rutgeerts P, et al. The safety of vedolizumab for ulcerative colitis and Crohn's disease. Gut 2016 Feb 18. doi: 10.1136/gutjnl-2015-311079. [Epub ahead of print]. 